\title{
Etapas fonológicas en flor y fruto en ají picante en condiciones de umbráculo, en la Universidad de los Llanos
}

Phonological stages in flower and fruit in hot pepper in greenhouse conditions at the Universidad de los Llanos

\author{
Carmen Carrillo Nydia ${ }^{1}$; Osorio Marulanda Diego Libardo ${ }^{2}$ Herrera Baquero C. A. ${ }^{3}$ \\ y Apolinar D. R. ${ }^{3}$ \\ ${ }^{1}$ Ingeniera Agrónoma, MSc., Universidad de los Llanos, Escuela de Ciencias \\ Agrícolas, Programa de Ingeniería Agronómica. ${ }^{2}$ Ingeniero Agrónomo Esp. \\ ${ }^{3}$ Ingeniero Agrónomo, Universidad de los Llanos \\ ncarmen@unillanos.edu.co
}

Recibido 30 de Mayo 2013, Aceptado 15 Julio 2013

\section{RESUMEN}

Se caracterizaron once accesiones de ají picante en la Universidad de los Llanos, situada en el kilómetro 12 vía a Puerto López, en el departamento del Meta, Colombia. Los genotipos fueron establecidos en bolsas plásticas negras (dimensiones $15 \times 15 \times 50 \mathrm{~cm}$ ) bajo umbráculo. Se evaluaron 16 plantas por genotipo en estado de floración y fructificación empleando la metodología descrita por IPGRI. Se determinó que la floración inicia cuando la planta empieza su ramificación, presentándose solitarias o en grupos de 2 o más en cada una de las axilas; el botón floral se abre dejando expuesta la corola, por un periodo de 15 días. En las accesiones Jalapeño, Pajarito y Cayenne la fructificación ocurrió a los 81, 102 y 103 días respectivamente. Sin embargo, las accesiones Conito y Yakaré, mostraron periodos de fructificación más largos, de 129 y 134 días.

Palabras clave: Descriptor morfológico, flor, fruto, ají.

\section{ABSTRACT}

Were characterized eleven accessions of hot chili under Universidad de Los Llanos environment condition, located twelve $\mathrm{km}$ road to Puerto Lopez. The genotypes were sowing in black plastic bags $(15 \times 15 \times 50 \mathrm{~cm})$ under artificial shade. Sixteen 
plants per genotype were evaluated at flowering and fruiting set stage, using IPGRI methodology. The flowering and tillering stage start at the same time, with one or two flowers per axil and the flower remains open during fifteen days. The fruit set of Jalapeño, Pajarito and Cayene, were at 81,102 and 103 days after sowing, respectively. However, the genotypes Conito and Yacaré, the fruit formation was at 129 and 134 days.

Keywords: Morphologic descriptor, flower, fruit, chili.

\section{INTRODUCCIÓN}

El ají (capsicum sp.) es originario de América tropical. El ají es considerado como el primer cultivar domesticado en el nuevo mundo, hecho que ha dado lugar a una gran cantidad de variedades adaptadas a los diferentes ambientes y a los requerimientos culturales donde se les cultiva. En particular, el principal valor nutritivo del ají lo constituye el alto contenido de vitaminas antioxidantes A, C y E. En los ajíes rojos son altas las cantidades de carotenoides que tienen la actividad provitamina A (Melgarejo, 2000).

En Colombia el ají picante es un cultivo relativamente reciente que comienza a expandirse por su buena rentabilidad, producción y posibilidad de exportación, siendo el color rojo del fruto un parámetro importante en la industria de las especias, el consumo de ají se ha incrementado y puede ser una fuente de vitaminas para la población mundial. Los mayores departamentos productores de ají fueron el Valle del Cauca, Santander, Antioquia y Bolívar. En el Valle geográfico del río Cauca, en los últimos años se han cultivado hasta 200 hectáreas de ajíes picantes para procesamiento y consumo local y de exportación. Colombia posee las condiciones climatológicas y la tecnología suficiente para llenar las expectativas crecientes que hay. Solo en ají Cayenne hay sembradas 6 mil toneladas. La proyección para los próximos años se estima en 1.000 hectáreas, con 25 mil toneladas de fruta fresca (Minagricultura, 2002).

Por sus diversos usos en la alimentación, en la parte artesanal, en la medicina humana, entre otros, el ají se perfila como uno de los cultivos más promisorios 
para la región, y también como generador de empleo en el área rural y un excelente producto agrícola como sustituto de cultivos ilícitos. Según lo estipulado en el plan 2020, el departamento del Meta se perfila como una de las zonas exportadoras; el área anual oscila entre 10 y 20 hectáreas con una producción de 40 toneladas (Secretaría de Agricultura, 2006).

El fruto de ají es una baya hueca, de superficie lisa y brillante, con forma y color muy variable y características del cultivar; el fruto se desarrolla a partir del gineceo de la flor y más específicamente a partir del ovario fecundado. La baya está constituida por un pericarpio grueso y jugoso y un tejido placentario al que se unen las semillas. El color del fruto maduro está determinado por pigmentos de carotenoides La mayoría de los frutos consumidos en fresco, tienen un pericarpio grueso, mientras que los usados secos o para molienda poseen un pericarpio delgado, con pocas capas de células y reducido contenido de agua (Popovsky y Paran, 2000).

La pungecia o sensación organoléptica de ardor; es el principal factor de determinación de la calidad y se debe a la presencia de uno o más de los 14 compuestos alcaloides conocidos como capsaicinoides, siendo la capsaicina $\left(\mathrm{C}_{16} \mathrm{H}_{27} \mathrm{O}_{3} \mathrm{~N}\right)$ el más importante entre estos; su contenido es mayor en la placenta y en el septo del fruto, en donde representa un $2.5 \%$ de la materia (Ligarreto et al., 2004).

La fenología agrícola se refiere a los fenómenos periódicos que presentan las plantas y su relación con las condiciones ambientales tales como: temperatura, luz, humedad, etc. Dichos fenómenos son por ejemplo: aparición de las hojas, floración, maduración. El conocimiento de las necesidades climáticas de una especie vegetal, permite una mejor elección del tipo de material a implementar en una zona o región, es decir que, la observación y cuantificación de los distintos fenómenos de los vegetales, que se relacionan con los elementos y factores climáticos, significan un paso en el conocimiento de las formas y metodologías que permitan un uso racional del medio ambiente en beneficio de la producción, (Nuez et al., 1996). 
En este sentido el objeto de esta investigación fue establecer y analizar las diferencias interespecíficas que muestren las accesiones en flor y en fruto, y efectuar la descripción de algunas características morfológicas y fenológicas en flor y fruto en accesiones de ají picante.

\section{MATERIALES Y MÉTODOS}

Se determinaron las etapas fenológicas en flor y fruto de once accesiones de ají picante bajo condiciones de un umbráculo en la Universidad de los Llanos sede Barcelona; se establecieron dieciséis plantas por accesión en bolsas plásticas negras; de dimensiones $15 \times 15 \times 50 \mathrm{~cm}$, estableciendo un total de ciento setenta y seis plantas. Se les suministró fertilización y riego de acuerdo a las exigencias determinadas en análisis de suelos.

Para determinar la etapa de botón floral y antesis, se escogieron doce botones florales al azar por accesión, los cuales se les hizo seguimiento diario. En la fenología del fruto se tomaron, doce botones florales por accesión (adicionales) y se les hizo seguimiento hasta la formación del fruto. Se tomó además el color, superficie y tamaño de la semilla, en diez semillas por accesión. Para la determinación de estas variables se empleó la metodología de lbid et al., (1995) y se presentan en la Tabla 1.

Tabla 1. Accesiones de ají picante empleadas en el estudio

\begin{tabular}{ccc}
\hline \multirow{2}{*}{ Accesiones } & \multicolumn{2}{c}{ Ajíes picantes } \\
\cline { 2 - 3 } & Especie & Variedad \\
\hline 1 & & Tabasco \\
2 & & Barrilito \\
3 & Capsicum & Yakaré \\
4 & frutescens & Pajarito \\
5 & & Mitu \\
6 & & Trompito \\
7 & & Conito \\
8 & Capsicum & Cayene \\
9 & annum & Jalapeño \\
10 & & Habanero largo \\
11 & & Habanero corto \\
\hline
\end{tabular}

Fuente: Los autores 


\section{Variables Dependientes:}

\section{Fenología}

- De la Inflorescencia: Días a la floración, número de flores por axila, posición de la flor, color de la corola, color de la mancha de la corola, forma de la mancha de la corola, color de las anteras, longitud de la antera $(\mathrm{mm})$, color del filamento, longitud del filamento $(\mathrm{mm})$, exserción del estigma.

- Del fruto: Días a la fructificación.

- Semilla: Color de la semilla, superficie de la semilla, tamaño de la semilla. diámetro de la semilla $(\mathrm{mm})$, peso de 1000 semillas $(\mathrm{g})$, número de semillas por fruto.

Variables Intervinientes: Condiciones climáticas.

\section{RESULTADOS Y DISCUSIÓN}

El desarrollo de la caracterización morfológica permitió una discriminación fácil y rápida entre fenotipos. Generalmente son caracteres altamente heredables y pueden ser fácilmente detectados a simple vista, se expresan igualmente en todos los ambientes. Además, pueden incluir un número limitado de caracteres adicionales que son deseables según las características de cada accesión.

La fenología de la inflorescencia (Gráfico 1) muestra cierto grado de precocidad para Jalapeño y Pajarito, con un promedio de 75.58 y 94.91 días a floración. Habanero largo y Trompito fueron los más tardíos con 128.58 y 147.16 días a floración respectivamente.

La floración inicia cuando la planta empieza su ramificación presentándose flores solitarias o en grupos de 2 o más en cada una de las axilas; el botón floral se presenta envuelto completamente en el cáliz, el cual se abre rápidamente hasta dejar expuesta la corola, durando un promedio de 15 días, desde el aparecimiento del primordio floral en las axilas de las ramas hasta la apertura total de la corola en 
concordancia con Melgarejo, (2000). En las Figuras 1, 2, 3 y 4 se muestra la flor de cuatro acciones de ají.

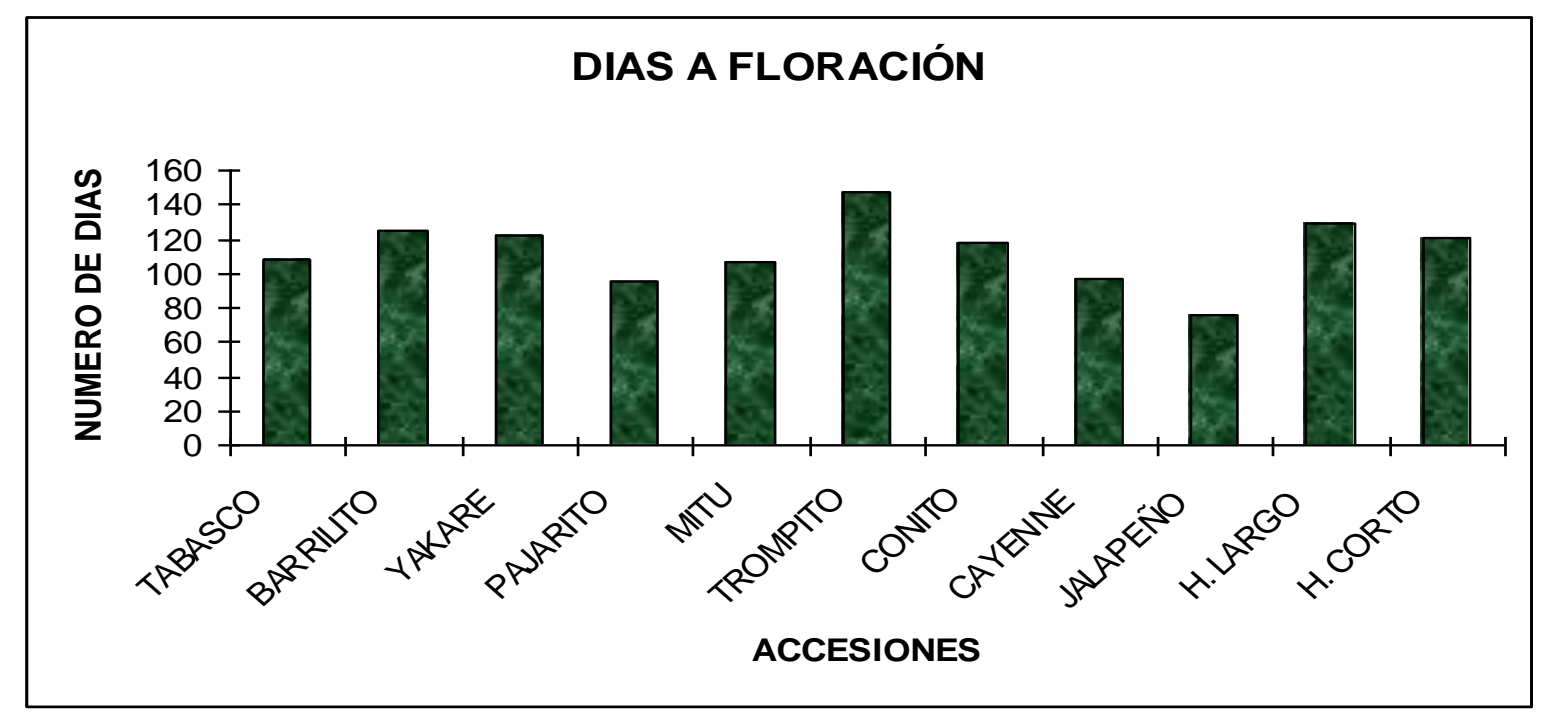

Gráfico1. Número de días a floración (Fuente: Los autores)

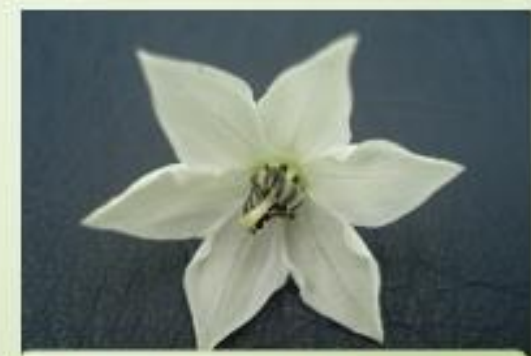

Figura1. Flor Jalapeño

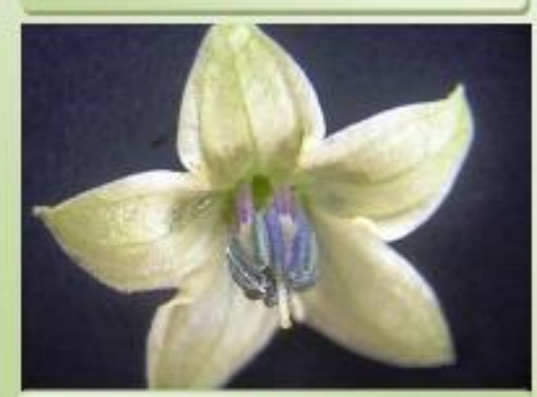

Figura 3. Flor Trompito



Figura 2. Flor Pajarito



Figura 4. Flor Habanero largo

Las flores comenzaron a abrir a las 4:30 a. m. y continuaron su apertura hasta las 7:10 a.m. con un máximo de las flores abiertas del 38.1\% (Barrilito, Yakaré y Trompito), seguido de un dato intermedio de $32.03 \%$ de flores abiertas $(\mathrm{H}$. corto, 
largo y Cayene) y un mínimo de $29.6 \%$ de flores abiertas (Tabasco, Pajarito y Jalapeño), después de las 7:10 a.m. no se registró antesis (Grafico 2).

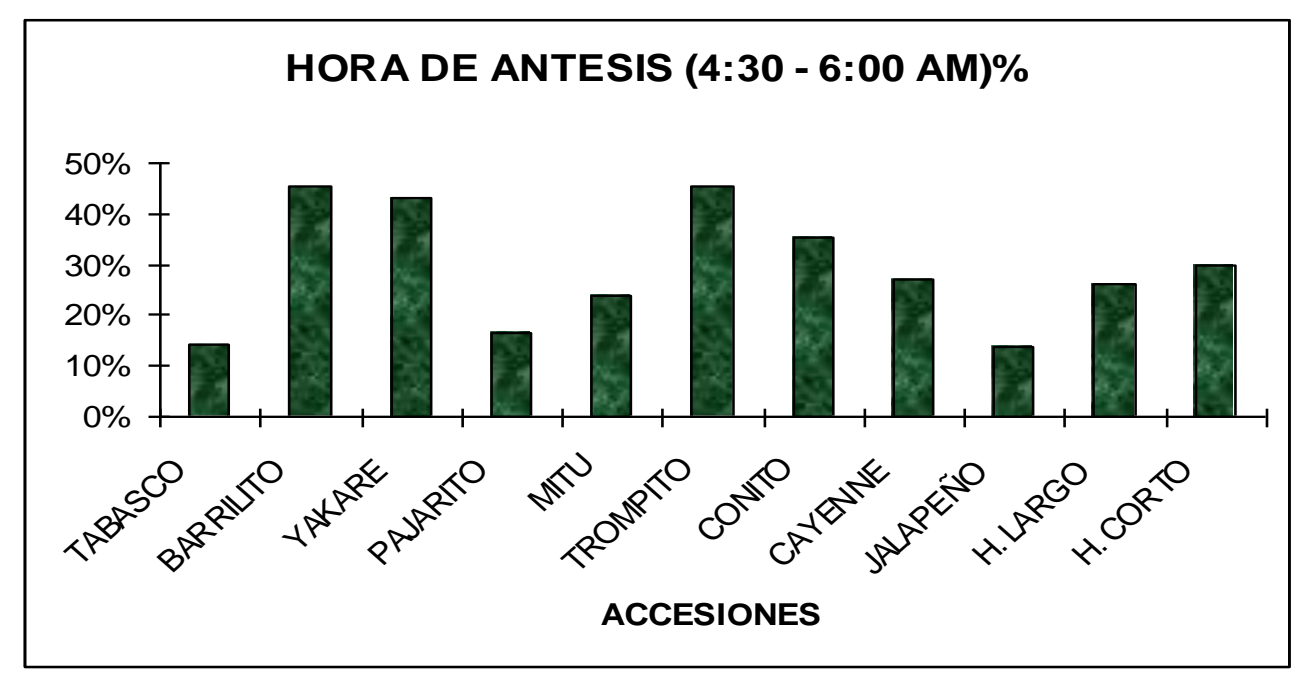

Gráfico 2. Hora de antesis

La fenología de fruto en las accesiones Jalapeño, Pajarito y Cayenne presentaron el menor número de días desde la siembra hasta la cosecha, con valores de 81 , 102 y 103 respectivamente. A su vez, las accesiones de periodos más largos fueron Trompito y Conito con 129 y 134 días a fructificación (Gráficos 3 y 4, Figuras 5,6 y 7 ).

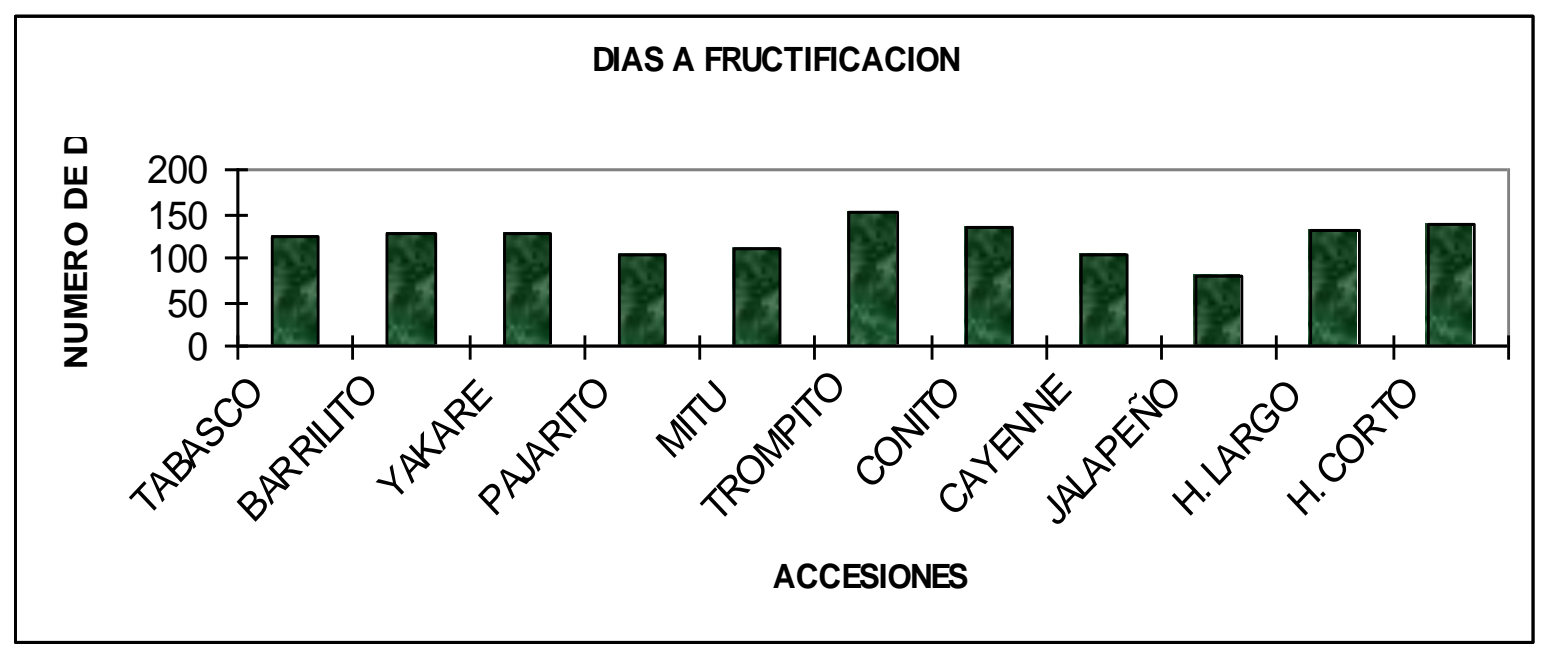

Gráfico 3. Días a fructificación 


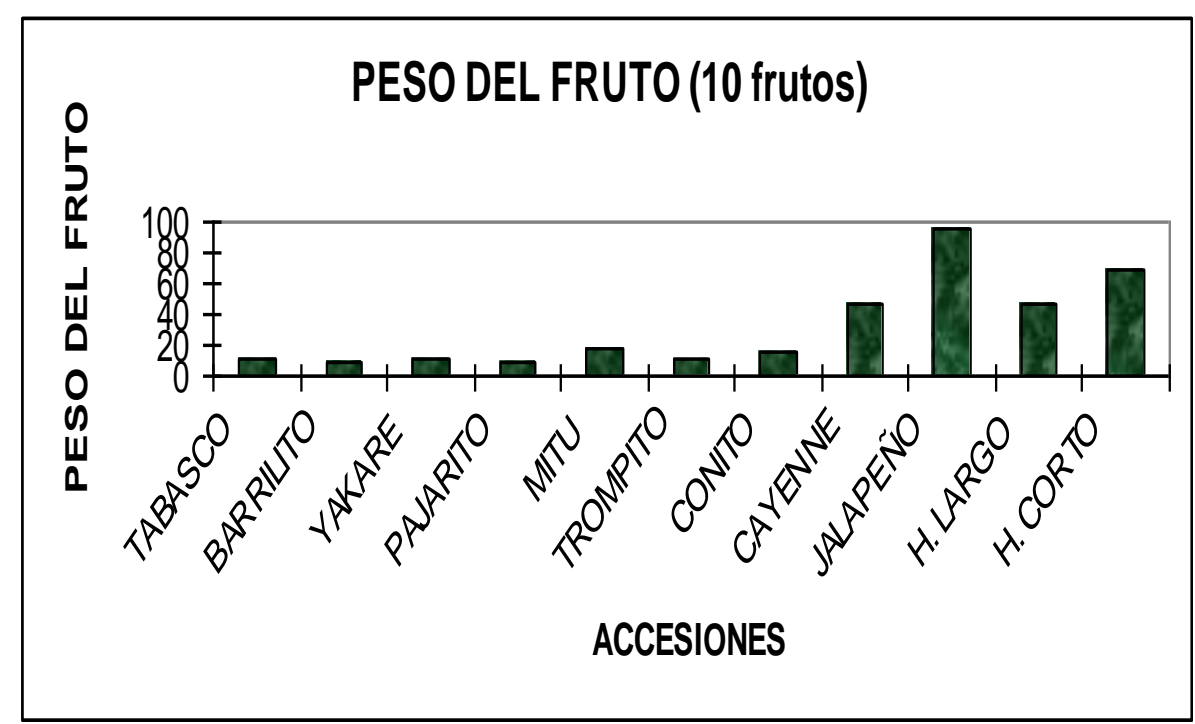

\section{Gráfico 4. Peso del fruto (gr.)}

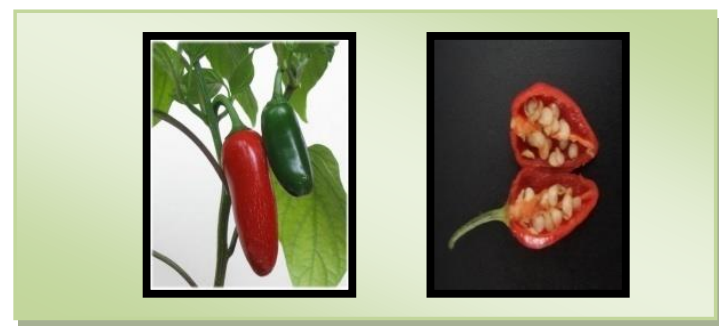

Figura 5. Frutos de Ají Jalapeño

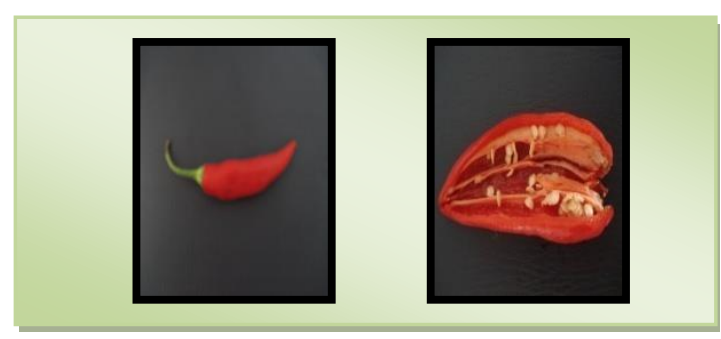

Figura 6. Frutos de Ají Pajarito

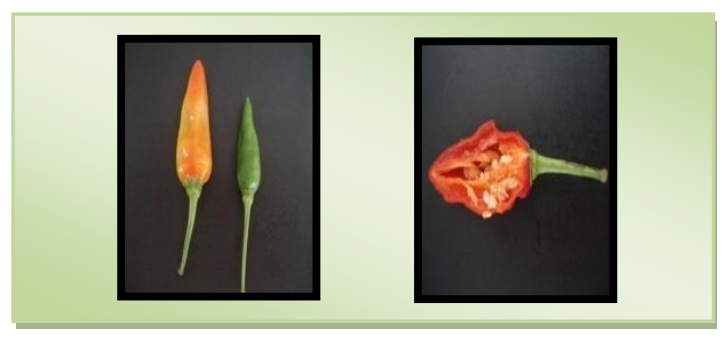

Figura 7. Frutos ají Cayene

Los datos obtenidos de las respectivas mediciones presentaron un rango de valores bastante extenso, como puede apreciarse en el Gráfico 3, se aprecian diferencias entre los materiales, encontrándose que la accesión Jalapeño peso 95,1 gr/fruto; mientras que la accesión Barrilito apenas obtuvo un peso de 8.3 gr/fruto. 
La fenología de la semilla en las accesiones Pajarito y Barrilito presentó el menor peso en gr de 1000, con valores de 4, 12 y 4,13 gr respectivamente. A su vez, los materiales que presentaron los mayores pesos, fueron la accesión Jalapeño y Cayenne con 8.45 y 7.51 gr respectivamente (Gráfico 5).



Gráfico 5. Peso de 1000 semillas

\section{CONCLUSIONES}

La floración inicia cuando la planta empieza su ramificación presentándose flores solitarias o en grupos de 2 o más en cada una de las axilas; el botón floral se presenta envuelto completamente en el cáliz, el cual se abre rápidamente hasta dejar expuesta la corola, durando un promedio de 15 días, desde el aparecimiento del primordio floral en las axilas de las ramas hasta la apertura total de la corola Los materiales de mayor precocidad a floración fueron Jalapeño y Pajarito, con un promedio de 75.58 y 94.91 días Habanero largo y Trompito fueron los más tardíos con 128.58 y 147.16 días respectivamente. 
Las flores comenzaron a abrir a las 4:30 a.m. y continuaron su apertura hasta las 7:10 a.m, con un máximo de flores abiertas del 38.1\% para Barrilito, Yakaré y trompito, seguido de $\mathrm{H}$. corto, largo y Cayene, con un $32.03 \%$ de flores abiertas.

Las accesiones más precoces fueron Jalapeño, Pajarito y Cayenne con 81,102 y 103 días desde la siembra hasta la cosecha. Trompito y Conito y fueron los más tardíos con 129 y 134 días a fructificación.

Las accesiones Pajarito y Barrilito presentaron el menor peso de semilla, con valores de 4,12 y 4,13 gr respectivamente. Los materiales que presentaron mayores pesos, fueron la accesión Jalapeño y Cayenne con 8.45 y 7.51 gr respectivamente.

\section{REFERENCIAS BIBLIOGRÁFICAS}

1. IPGRI, AVRDC, CATIE. Descriptores para Capsicum (Capsicum spp.). Instituto Internacional de Recursos Fitogenéticos, Roma, Italia; Centro Asiático para el Desarrollo y la Investigación relativos a los Vegetales, Taipei, Taiwán y Centro Agronómico Tropical de Investigación y Enseñanza, Turrialba, Costa Rica. ISBN 92-9043-216-0 IPGRI AVRDC CATIE Via delle Sette Chiese 142 P.O. Box 205 Apartado 7400145 Roma Taipei Turrialba Italia Taiwán Costa Rica (C) Internacional Plant Genetic Resources Institute 1995 Descriptores para Capsicum. p 60. 1995.

2. Ligarreto G, A. Espinosa N., Méndez M, A. Recursos genéticos y cultivo de ají y pimentón, Capsicum Spp; Universidad Nacional de Colombia Facultad de Agronomía, Sede Bogotá, 1a ed. 2004

3. Melgarejo L. M. Informe Técnico Final. Caracterización morfológica, bioquímica y molecular de especies promisorias de la Amazonia colombiana pertenecientes al género Capsicum sp. para su conservación y uso. Bogotá. Instituto SINCHI. p 120. 2000.

4. Ministerio de Agricultura. Anuario estadístico. Desarrollo Rural, 1997 y 2002.

5. Nuez F., Gil Ortega R., Costa J. El cultivo de pimientos, chiles y ajíes. Ediciones Mundi-Prensa Madrid-España. 586 p. 1996.

6. Popovsky S., Paran I. Molecular genetics of the y locus in pepper. Its relation to capsanthin-capsorubin synthase and to fruit color. Theorical Applicate Genetic. 101: 86-87. 2000.

7. Secretaría de Agricultura. Gobernación del Departamento del Meta. Anuario Estadístico, Unidad de Estadística y Estudios. 2006. 